\title{
МАТЕМАТИКАИ МЕХАНИКА
}

DOI: https://doi.org/10.15688/mpcm.jvolsu.2018.3.1

УДК 517.983

ББК 22.162

\section{ОБРАТИМОСТЬ ИНТЕГРАЛЬНЫХ ОПЕРАТОРОВ С ОДНОРОДНЫМИ ЯДРАМИ КОМПАКТНОГО ТИПА НА ГРУППЕ ГЕЙЗЕНБЕРГА}

\section{Виктор Владимирович Денисенко}

Аспирант кафедры алгебры и дискретной математики,

Южный федеральный университет, институт математики, механики и компьютерных наук им. И.И. Воровича

vct.dns@gmail.com

ул. Мильчакова, 8a, 344090 г. Ростов-на-Дону, Российская Федерация

\section{Владимир Михайлович Деундяк}

Кандидат физико-математических наук, доцент кафедры алгебры

и дискретной математики,

Южный федеральный университет, институт математики, механики

и компьютерных наук им. И.И. Воровича

vl.deundyak@gmail.com

ул. Мильчакова, 8a, 344090 г. Ростов-на-Дону, Российская Федерация;

Старший научный сотрудник,

ФГНУ НИИ «Спецвузавтоматика»

пер. Газетный, 51, 344002 г. Ростов-на-Дону, Российская Федерация

Аннотация. Рассматривается группа Гейзенберга $\mathbb{H}_{n}$ с нормой Кораньи. В пространстве $L_{p}\left(\mathbb{H}_{n}\right), 1<p<\infty$, вводятся интегральные операторы с однородными ядрами компактного типа. Для банаховой алгебры с единицей $\mathfrak{V}_{p}^{+}\left(\mathbb{H}_{n}\right)$, порожденной операторами такого типа, строится символическое исчисление, и в его терминах формулируются необходимые и достаточные условия обратимости операторов из $\mathfrak{V}_{p}^{+}\left(\mathbb{H}_{n}\right)$. Эти результаты получены с помощью перехода к сферической системе координат на группе Гейзенберга $\mathbb{H}_{n}$ и сверточного представления алгебры $\mathfrak{V}_{p}^{+}\left(\mathbb{H}_{n}\right)$.

Ключевые слова: группа Гейзенберга, линейные интегральные операторы, операторы с однородными ядрами, сверточное представление, символическое исчисление, обратимость операторов.

ISSN 2587-6325. Математ. физика и компьютер. моделирование. 2018. T. 21. № 3 


\section{Введение}

В пространстве $L_{p}\left(\mathbb{R}^{n}\right), 1<p<\infty$, рассмотрим оператор растяжения $\vartheta_{\alpha}$, определяемый равенством $\left(\vartheta_{\alpha} f\right)(x)=f(x / \alpha), \alpha>0$. В [8] введен и изучен класс однородных операторов, коммутирующих со всеми операторами $\vartheta_{\alpha}$. Этому классу принадлежат интегральные операторы с однородными ядрами, которые в настоящий момент хорошо изучены (см., например, $[1 ; 4 ; 14]$ и цитированные там источники). Для таких операторов получены условия ограниченности и обратимости, для операторов с различными классами коэффициентов получены условия фредгольмовости и формулы для вычисления индекса. В [5] рассмотрены интегральные операторы с анизотропно однородными ядрами компактного типа, а в [13] - однородные операторы в гильбертовых модулях.

В связи с развитием в последнее время некоммутативного гармонического анализа и его применением в различных областях науки и техники $[12 ; 15 ; 16]$ представляется актуальным распространить теорию операторов с однородными ядрами с группы $\mathbb{R}^{n}$ на некоммутативные группы. В работе [3] введен класс интегральных операторов с однородными ядрами на группе Гейзенберга, для которых получены условия ограниченности и регулярности. В настоящей статье на группе Гейзенберга вводится новый класс однородных ядер компактного типа и для унитализации банаховой алгебры $\mathfrak{V}_{p}^{+}\left(\mathbb{H}_{n}\right)$, порожденной интегральными операторами с ядрами такого типа, строится символическое исчисление, в терминах которого формулируется критерий обратимости.

\section{1. Группа Гейзенберга}

В этом разделе приведем в удобном для дальнейшего виде необходимые сведения о группе Гейзенберга (см., например, [16, с. 209-212]). Пусть $\mathbb{N}-$ множество натуральных чисел; $\mathbb{R}$ - аддитивная группа вещественных чисел; $\mathbb{R}_{+}-$мультипликативная группа положительных чисел; $\mathbb{C}^{n}-n$-мерное унитарное пространство со скалярным произведением

$$
z \cdot w=\sum_{k=1}^{n} z_{k} \bar{w}_{k}, \quad z, w \in \mathbb{C}^{n}
$$

и нормой

$$
|z|=\left(\sum_{k=1}^{n}\left|z_{k}\right|^{2}\right)^{\frac{1}{2}}, \quad z \in \mathbb{C}^{n} .
$$

Группой Гейзенберга называется множество $\mathbb{H}_{n}=\mathbb{C}^{n} \times \mathbb{R}$ с бинарной операцией

$$
(z, a)(w, b)=(z+w, a+b+2 \operatorname{Im}(z \cdot w)), \quad(z, a),(w, b) \in \mathbb{H}_{n} .
$$

Нейтральный элемент группы имеет вид $(\mathbf{0}, 0)$, где $\mathbf{0}-$ нулевой вектор пространства $\mathbb{C}^{n}$, а обратный элемент вычисляется по формуле $(z, a)^{-1}=(-z,-a)$. Заметим, что в некоторых источниках групповая операция может определяться иначе (см., например, $[11$, c. 13]). Кроме того, следует отметить, что группу Гейзенберга часто определяют как подгруппу группы квадратных верхнетреугольных матриц размера $n+2$ следующего вида:

$$
\left(\begin{array}{ccc}
1 & a & c \\
0 & E_{n} & b^{T} \\
0 & 0 & 1
\end{array}\right)
$$


где $E_{n}-$ единичная матрица порядка $n, a$ и $b-$ вектор-строки из $\mathbb{R}^{n}$, $b \mapsto b^{T}-$ операция транспонирования, $c \in \mathbb{R}$. Все упомянутые подходы приводят к изоморфным группам.

Для любого $r \in \mathbb{R}_{+}$существует автоморфизм $\delta_{r}: \mathbb{H}_{n} \rightarrow \mathbb{H}_{n}$, определяемый формулой

$$
\delta_{r}(z, a)=\left(r z, r^{2} a\right), \quad(z, a) \in \mathbb{H}_{n} .
$$

Автоморфизм $\delta_{r}$ называют растяжением. Группа автоморфизмов $\left\{\delta_{r}\right\}_{r \in \mathbb{R}_{+}}$с операцией композиции изоморфна группе $\mathbb{R}_{+}$, при этом $\delta_{r \rho}=\delta_{r} \delta_{\rho}$ и $\delta_{r}^{-1}=\delta_{\frac{1}{r}}$ для любых $r, \rho \in \mathbb{R}_{+}$.

Снабдим группу $\mathbb{H}_{n}$ нормой Кораньи:

$$
\|(z, a)\|=\left(|z|^{4}+a^{2}\right)^{\frac{1}{4}}, \quad(z, a) \in \mathbb{H}_{n},
$$

которая удовлетворяет условию однородности:

$$
\forall x \in \mathbb{H}_{n}, r \in \mathbb{R}_{+}: \quad\left\|\delta_{r}(x)\right\|=r\|x\|
$$

Норма Кораньи позволяет определить на группе Гейзенберга единичную сферу

$$
\mathbb{S}_{n}=\left\{x \in \mathbb{H}_{n}:\|x\|=1\right\}
$$

с центром в точке $(\mathbf{0}, 0) \in \mathbb{H}_{n}$, которая гомеоморфна стандартной $2 n$-мерной сфере в пространстве $\mathbb{R}^{2 n+1}$.

На группе Гейзенберга преобразование декартовых координат $x \in \mathbb{H}_{n} \backslash\{(\mathbf{0}, 0)\}$ в сферические $(r, s) \in \mathbb{R}_{+} \times \mathbb{S}_{n}$ определяется по формулам

$$
r=\|x\|, \quad s=\delta_{\|x\|}^{-1}(x),
$$

при этом для любой определенной на $\mathbb{H}_{n}$ интегрируемой функции $f$

$$
\int_{\mathbb{H}_{n}} f(x) d x=\int_{\mathbb{S}_{n}} \int_{0}^{\infty} f\left(\delta_{r}(s)\right) r^{2 n+1} d r d s .
$$

Стандартная мера Лебега на группе $\mathbb{R}^{2 n+1}$ задает биинвариантную меру Хаара на группе Гейзенберга $\mathbb{H}_{n}[16$, с. 192]. Ниже мы будем рассматривать лебеговы банаховы пространства $L_{p}\left(\mathbb{H}_{n}\right)$, где $1<p<\infty$ и $L_{\infty}\left(\mathbb{S}_{n}\right)$. Кроме того, далее понадобится естественный аналог пространства со смешанной нормой $L_{(1, \infty)}\left(\mathbb{R}^{n} \times \mathbb{R}^{n}\right.$ ) (см., например, $\left[2\right.$, с. 8-11]), а именно весовое пространство $L_{(1, \infty)}\left(\mathbb{H}_{n} \times \mathbb{H}_{n}, \omega_{1} \otimes \omega_{2}\right)$, то есть пространство, состоящее из определенных на $\mathbb{H}_{n} \times \mathbb{H}_{n}$ измеримых функций $f$, для которых конечна норма

$$
\|f\|_{L_{(1, \infty)}\left(\mathbb{H}_{n} \times \mathbb{H}_{n}, \omega_{1} \otimes \omega_{2}\right)}=\underset{y \in \mathbb{H}_{n}}{\operatorname{ess~sup}}\left|\omega_{2}(y) \int_{\mathbb{H}_{n}}\right| f(x, y)\left|\omega_{1}(x) d x\right|,
$$

где $\omega_{1}, \omega_{2}-$ весовые функции на $\mathbb{H}_{n}$.

ISSN 2587-6325. Математ. физика и компьютер. моделирование. 2018. Т. 21. № 3 


\section{2. Интегральные операторы с однородными ядрами на группе $\mathbb{H}_{n}$}

Пусть $1<p, p^{\prime}<\infty, 1 / p+1 / p^{\prime}=1$. Определенную на $\mathbb{H}_{n} \times \mathbb{H}_{n}$ функцию $k$ назовем однородной степени $m$, если

$$
\forall \gamma \in \mathbb{R}_{+}, \quad \forall x, y \in \mathbb{H}_{n}: \quad k\left(\delta_{\gamma}(x), \delta_{\gamma}(y)\right)=\gamma^{m} k(x, y) .
$$

Однородную степени $(-2 n-2)$ измеримую функцию $k$ отнесем к классу $\mathcal{M}_{p}\left(\mathbb{H}_{n}\right)$, если

$$
\begin{aligned}
& \varkappa_{1}(k)=\underset{\sigma \in \mathbb{S}_{n}}{\operatorname{esssup}}\left|\int_{\mathbb{H}_{n}}\right| k(x, \sigma)|\| x|^{-\frac{2 n+2}{p^{\prime}}} d x \mid<\infty, \\
& \varkappa_{2}(k)=\underset{s \in \mathbb{S}_{n}}{\operatorname{ess} \sup _{n}}\left|\int_{\mathbb{H}_{n}}\right| k(s, y)|\| y|^{-\frac{2 n+2}{p}} d y \mid<\infty .
\end{aligned}
$$

Лемма 1. $\mathcal{M}_{p}\left(\mathbb{H}_{n}\right)$ - банахово пространство с нормой

$$
\|k\|_{\mathcal{M}_{p}\left(\mathbb{H}_{n}\right)}=\max \left(\varkappa_{1}(k), \varkappa_{2}(k)\right) .
$$

Доказательство. Для любой функции $k \in \mathcal{M}_{p}\left(\mathbb{H}_{n}\right)$ и соответствующих чисел $\varkappa_{1}(k), \varkappa_{2}(k)$ справедливы равенства

$$
\begin{aligned}
& \varkappa_{1}(k)=\underset{y \in \mathbb{H}_{n}}{\operatorname{ess} \sup _{1}}\left|\|y\|^{\frac{2 n+2}{p^{\prime}}} \int_{\mathbb{H}_{n}}\right| k(x, y)\left|\|x\|^{-\frac{2 n+2}{p^{\prime}}} d x\right|, \\
& \varkappa_{2}(k)=\underset{x \in \mathbb{H}_{n}}{\operatorname{ess} \sup _{n}}\left|\|x\|^{\frac{2 n+2}{p}} \int_{\mathbb{H}_{n}}\right| k(x, y)\left|\|y\|^{-\frac{2 n+2}{p}} d y\right| .
\end{aligned}
$$

Действительно, используя замену $x \mapsto \delta_{\|y\|}(x), d x \mapsto\|y\|^{2 n+2} d x$, а также воспользовавшись свойством однородности нормы Кораньи (см. (1)) и свойством однородности функции $k$ (см. (5)), легко убедиться, что

$$
\forall y \in \mathbb{H}_{n}: \quad\|y\|^{\frac{2 n+2}{p^{\prime}}} \int_{\mathbb{H}_{n}}|k(x, y)|\|x\|^{-\frac{2 n+2}{p^{\prime}}} d x=\int_{\mathbb{H}_{n}}\left|k\left(x, \delta_{\|y\|}^{-1}(y)\right)\right|\|x\|^{-\frac{2 n+2}{p^{\prime}}} d x .
$$

Далее, замечая, что

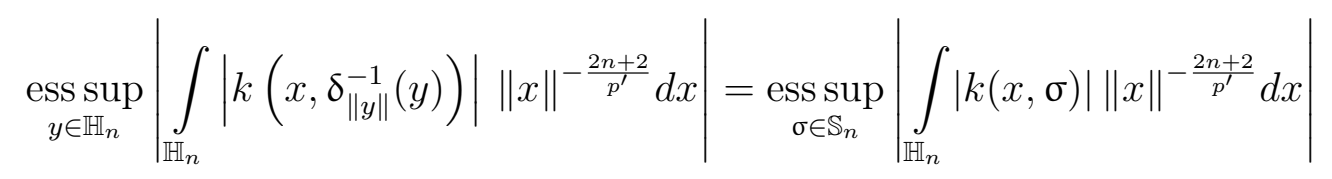

и учитывая (6), получаем (9). Выполнение равенства (10) доказывается аналогично.

Множество определенных на $\mathbb{H}_{n} \times \mathbb{H}_{n}$ измеримых функций $k$, удовлетворяющих условию $\varkappa_{1}(k)<\infty$ (см. (9)), является банаховым пространством

$$
L_{(1, \infty)}\left(\mathbb{H}_{n} \times \mathbb{H}_{n},\|x\|^{-\frac{2 n+2}{p^{\prime}}} \otimes\|y\|^{\frac{2 n+2}{p^{\prime}}}\right),
$$


(см. (4)). Подпространство этого пространства, порожденное однородными степени $(-2 n-$ $-2)$ функциями, обозначим $\mathcal{M}_{p}^{1}\left(\mathbb{H}_{n}\right)$. Норма в данном пространстве задается равенством

$$
\|k\|_{\mathcal{M}_{p}^{1}\left(\mathbb{H}_{n}\right)}=\varkappa_{1}(k) .
$$

Множество определенных на $\mathbb{H}_{n} \times \mathbb{H}_{n}$ измеримых функций $k$, удовлетворяющих условию $\varkappa_{2}(k)<\infty$ (см. (10)), обозначим $\mathcal{M}_{p}^{0}\left(\mathbb{H}_{n}\right)$. Каждой функции $k \in \mathcal{M}_{p}^{0}\left(\mathbb{H}_{n}\right)$ сопоставим функцию $\tilde{k}(x, y)=k(y, x)$, которая, как несложно заметить, удовлетворяет условию

$$
\underset{y \in \mathbb{H}_{n}}{\operatorname{ess} \sup _{1}}\left|\|y\|^{\frac{2 n+2}{p}} \int_{\mathbb{H}_{n}}\right| \tilde{k}(x, y)\left|\|x\|^{-\frac{2 n+2}{p}} d x\right|<\infty .
$$

Множество определенных на $\mathbb{H}_{n} \times \mathbb{H}_{n}$ измеримых функций $\tilde{k}$, для которых справедливо это условие, является банаховым пространством

$$
L_{(1, \infty)}\left(\mathbb{H}_{n} \times \mathbb{H}_{n},\|x\|^{-\frac{2 n+2}{p}} \otimes\|y\|^{\frac{2 n+2}{p}}\right),
$$

откуда следует, что $\mathcal{M}_{p}^{0}\left(\mathbb{H}_{n}\right)$ также является банаховым пространством. Подпространство пространства $\mathcal{M}_{p}^{0}\left(\mathbb{H}_{n}\right)$, порожденное однородными степени $(-2 n-2)$ функциями, обозначим $\mathcal{M}_{p}^{2}\left(\mathbb{H}_{n}\right)$. Норма в данном пространстве определяется равенством

$$
\|k\|_{\mathcal{M}_{p}^{2}\left(\mathbb{H}_{n}\right)}=\varkappa_{2}(k) \text {. }
$$

Банаховы пространства $\mathcal{M}_{p}^{1}\left(\mathbb{H}_{n}\right)$ и $\mathcal{M}_{p}^{2}\left(\mathbb{H}_{n}\right)$ образуют банахову пару, а их пересечение

$$
\mathcal{M}_{p}\left(\mathbb{H}_{n}\right)=\mathcal{M}_{p}^{1}\left(\mathbb{H}_{n}\right) \bigcap \mathcal{M}_{p}^{2}\left(\mathbb{H}_{n}\right)
$$

является банаховым пространством с нормой (8) (см. [6, с. 20]). Лемма доказана.

В пространстве $L_{p}\left(\mathbb{H}_{n}\right)$ рассмотрим интегральный оператор

$$
\left(K_{k} f\right)(x)=\int_{\mathbb{H}_{n}} k(x, y) f(y) d y, \quad k \in \mathcal{M}_{p}\left(\mathbb{H}_{n}\right) .
$$

Из теоремы 1 работы [3] вытекает следующее утверждение.

Теорема 1. Oператор $K_{k}$ вида (11) ограничен в $L_{p}\left(\mathbb{H}_{n}\right)$.

Для произвольного банахова пространства $\mathfrak{X}$ через $\mathcal{L}(\mathfrak{X})$ будем обозначать банахову алгебру всех линейных, ограниченных в $\mathfrak{X}$, операторов. Замкнутую подалгебру банаховой алгебры $\mathcal{L}\left(L_{p}\left(\mathbb{H}_{n}\right)\right)$, порожденную интегральными операторами вида $(11)$, обозначим $\operatorname{Op}\left(\mathcal{M}_{p}\left(\mathbb{H}_{n}\right)\right)$.

\section{3. Операторы из $\mathrm{Op}\left(\mathcal{M}_{p}\left(\mathbb{H}_{n}\right)\right)$ в сферической системе координат}

Для того чтобы в алгебре $\mathrm{Op}\left(\mathcal{M}_{p}\left(\mathbb{H}_{n}\right)\right)$ выделить подалгебру операторов с однородными ядрами компактного типа, рассмотрим операторы из $\operatorname{Op}\left(\mathcal{M}_{p}\left(\mathbb{H}_{n}\right)\right)$ в сферической системе координат. Преобразование декартовых координат в сферические на группе $\mathbb{H}_{n}$ индуцирует изометрический изоморфизм

$$
Q: L_{p}\left(\mathbb{H}_{n}\right) \rightarrow L_{p}\left(\mathbb{R}_{+} \times \mathbb{S}_{n}, r^{2 n+1} \otimes 1\right)
$$


(см. (2)-(3)), который определяется по формуле

$$
(Q f)(r, s)=f\left(\delta_{r}(s)\right), \quad r \in \mathbb{R}_{+}, s \in \mathbb{S}_{n} .
$$

Определенную на $\mathbb{R}_{+} \times \mathbb{R}_{+} \times \mathbb{S}_{n} \times \mathbb{S}_{n}$ измеримую функцию $l$, удовлетворяющую условию

$$
\forall \gamma, r, \rho \in \mathbb{R}_{+}, \quad \forall s, \sigma \in \mathbb{S}_{n}: \quad l(\gamma r, \gamma \rho, s, \sigma)=\gamma^{-1} l(r, \rho, s, \sigma),
$$

отнесем к классу $\mathcal{M}_{p}^{\prime}\left(\mathbb{H}_{n}\right)$, если

$$
\begin{aligned}
& \varkappa_{1}^{\prime}(l)=\underset{\sigma \in \mathbb{S}_{n}}{\operatorname{ess} \sup _{0}}\left|\int_{\mathbb{S}_{n}} \int_{0}^{\infty}\right| l(r, 1, s, \sigma)\left|r^{\frac{2 n+2}{p}-1} d r d s\right|<\infty, \\
& \varkappa_{2}^{\prime}(l)=\underset{s \in \mathbb{S}_{n}}{\operatorname{ess} \sup _{0}}\left|\int_{\mathbb{S}_{n}} \int_{0}^{\infty}\right| l(1, \rho, s, \sigma)\left|\rho^{-\frac{2 n+2}{p}} d \rho d \sigma\right|<\infty .
\end{aligned}
$$

Аналогично лемме 1 доказывается, что $\mathcal{M}_{p}^{\prime}\left(\mathbb{H}_{n}\right)$ - банахово пространство с нормой

$$
\|l\|_{\mathcal{M}_{p}^{\prime}\left(\mathbb{H}_{n}\right)}=\max \left(\varkappa_{1}^{\prime}(l), \varkappa_{2}^{\prime}(l)\right) .
$$

Изометрический изоморфизм $\widetilde{Q}: \mathcal{M}_{p}\left(\mathbb{H}_{n}\right) \rightarrow \mathcal{M}_{p}^{\prime}\left(\mathbb{H}_{n}\right)$ определим по формуле

$$
(\widetilde{Q} k)(r, s, \rho, \sigma)=\rho^{2 n+1} k\left(\delta_{r}(s), \delta_{\rho}(\sigma)\right), \quad r, \rho \in \mathbb{R}_{+}, s, \sigma \in \mathbb{S}_{n}
$$

В пространстве $L_{p}\left(\mathbb{R}_{+} \times \mathbb{S}_{n}, r^{2 n+1} \otimes 1\right)$ рассмотрим интегральный оператор

$$
\left(L_{l} f\right)(r, s)=\int_{\mathbb{S}_{n}} \int_{0}^{\infty} l(r, \rho, s, \sigma) f(\rho, \sigma) d \rho d \sigma, \quad l \in \mathcal{M}_{p}^{\prime}\left(\mathbb{H}_{n}\right) .
$$

Для любого изоморфизма произвольных банаховых пространств $T: \mathfrak{X}_{1} \rightarrow \mathfrak{X}_{2}$ пространственный изоморфизм подобия банаховых алгебр $\widehat{T}: \mathcal{L}\left(\mathfrak{X}_{1}\right) \rightarrow \mathcal{L}\left(\mathfrak{X}_{2}\right)$ определяется с помощью равенства

$$
\widehat{T}(\Theta)=T \Theta T^{-1}, \quad \Theta \in \mathcal{L}\left(\mathfrak{X}_{1}\right) .
$$

Непосредственными выкладками доказывается, что для отображения $\widetilde{Q}$ (см. (13)) и изоморфизма подобия

$$
\widehat{Q}: \mathcal{L}\left(L_{p}\left(\mathbb{H}_{n}\right)\right) \rightarrow \mathcal{L}\left(L_{p}\left(\mathbb{R}_{+} \times \mathbb{S}_{n}, r^{2 n+1} \otimes 1\right)\right)
$$

определяемого с помощью изоморфизма $Q$ (см. (12)), справедливо соотношение

$$
\forall k \in \mathcal{M}_{p}\left(\mathbb{H}_{n}\right): \quad \widehat{Q}\left(K_{k}\right)=L_{\widetilde{Q}(k)},
$$

откуда, в частности, следует, что в силу теоремы 1 оператор вида (14) ограничен в пространстве $L_{p}\left(\mathbb{R}_{+} \times \mathbb{S}_{n}, r^{2 n+1} \otimes 1\right)$. Замкнутую подалгебру банаховой алгебры $\mathcal{L}\left(L_{p}\left(\mathbb{R}_{+} \times\right.\right.$ $\left.\left.\times \mathbb{S}_{n}, r^{2 n+1} \otimes 1\right)\right)$, порожденную интегральными операторами вида $(14)$, обозначим $\mathrm{Op}\left(\mathcal{M}_{p}^{\prime}\left(\mathbb{H}_{n}\right)\right)$, тогда

$$
\widehat{Q}\left(\mathrm{Op}\left(\mathcal{M}_{p}\left(\mathbb{H}_{n}\right)\right)\right)=\operatorname{Op}\left(\mathcal{M}_{p}^{\prime}\left(\mathbb{H}_{n}\right)\right)
$$

10 В.В. Денисенко, В.М. Деундяк. Обратимость интегральных операторов на группе Гейзенберга 


\section{4. Операторы с однородными ядрами компактного типа на группе $\mathbb{H}_{n}$}

Будем полагать, что определенная на $\mathbb{R}_{+} \times \mathbb{R}_{+}$измеримая функция $a$, удовлетворяющая условию

$$
\forall \gamma, r, \rho \in \mathbb{R}_{+}: \quad a(\gamma r, \gamma \rho)=\gamma^{-1} a(r, \rho),
$$

принадлежит классу $\mathcal{A}_{p}\left(\mathbb{R}_{+}, r^{2 n+1}\right)$, если

$$
\alpha(a)=\int_{0}^{\infty}|a(r, 1)| r^{\frac{2 n+2}{p}-1} d r=\int_{0}^{\infty}|a(1, \rho)| \rho^{-\frac{2 n+2}{p}} d \rho<\infty .
$$

Нетрудно доказать, что $\mathcal{A}_{p}\left(\mathbb{R}_{+}, r^{2 n+1}\right)$ - банахово пространство с нормой

$$
\|a\|_{\mathcal{A}_{p}\left(\mathbb{R}_{+}, r^{2 n+1}\right)}=\alpha(a) .
$$

В пространстве $L_{p}\left(\mathbb{R}_{+}, r^{2 n+1}\right)$ рассмотрим оператор мультипликативной свертки

$$
\left(A_{a} f\right)(r)=\int_{0}^{\infty} a(r, \rho) f(\rho) d \rho, \quad a \in \mathcal{A}_{p}\left(\mathbb{R}_{+}, r^{2 n+1}\right) .
$$

Известно, что оператор $A_{a}$ вида $(20)$, ограничен в $L_{p}\left(\mathbb{R}_{+}, r^{2 n+1}\right)[14$, с. 53]. Замкнутую подалгебру банаховой алгебры $\mathcal{L}\left(L_{p}\left(\mathbb{R}_{+}, r^{2 n+1}\right)\right)$, порожденную интегральными операторами вида (20), обозначим $\mathcal{V}_{p}\left(\mathbb{R}_{+}, r^{2 n+1}\right)$.

Рассмотрим алгебраическое тензорное произведение

$\mathcal{A}_{p}\left(\mathbb{R}_{+}, r^{2 n+1}\right) \odot L_{\infty}\left(\mathbb{S}_{n} \times \mathbb{S}_{n}\right)=\left\{\sum_{i=1}^{j} a_{i} b_{i}: a_{i} \in \mathcal{A}_{p}\left(\mathbb{R}_{+}, r^{2 n+1}\right), b_{i} \in L_{\infty}\left(\mathbb{S}_{n} \times \mathbb{S}_{n}\right), j \in \mathbb{N}\right\}$,

вложенное в $\mathcal{M}_{p}^{\prime}\left(\mathbb{H}_{n}\right)$. Через $\mathcal{C}_{p}^{\prime}\left(\mathbb{H}_{n}\right)$ обозначим замыкание $\mathcal{A}_{p}\left(\mathbb{R}_{+}, r^{2 n+1}\right) \odot L_{\infty}\left(\mathbb{S}_{n} \times \mathbb{S}_{n}\right)$ в $\mathcal{M}_{p}^{\prime}\left(\mathbb{H}_{n}\right)$. Пусть

$$
\mathcal{C}_{p}\left(\mathbb{H}_{n}\right)=\widetilde{Q}^{-1}\left(\mathcal{C}_{p}^{\prime}\left(\mathbb{H}_{n}\right)\right) .
$$

В силу того что интегральные операторы с ядрами из $L_{\infty}\left(\mathbb{S}_{n} \times \mathbb{S}_{n}\right)$ компактны в пространстве $L_{p}\left(\mathbb{S}_{n}\right)$, ядра из $\mathcal{C}_{p}\left(\mathbb{H}_{n}\right)$ будем, аналогично работе [4], называть ядрами компактного типа.

Замкнутую подалгебру банаховой алгебры $\mathrm{Op}\left(\mathcal{M}_{p}\left(\mathbb{H}_{n}\right)\right)$, порожденную интегральными операторами $(11)$ с ядрами из $\mathcal{C}_{p}\left(\mathbb{H}_{n}\right)$, обозначим $\mathfrak{V}_{p}\left(\mathbb{H}_{n}\right)$. Для произвольной банаховой алгебры $\mathfrak{A}$ через $\mathfrak{A}^{+}$обозначим ее унитализацию. В частности, $\mathfrak{V}_{p}^{+}\left(\mathbb{H}_{n}\right)$ унитализация $\mathfrak{V}_{p}\left(\mathbb{H}_{n}\right)$.

Из равенства (17) следует, что алгебра $\mathfrak{V}_{p}^{\prime}\left(\mathbb{H}_{n}\right)=\widehat{Q}\left(\mathfrak{V}_{p}\left(\mathbb{H}_{n}\right)\right)$ совпадает с замкнутой подалгеброй банаховой алгебры $\mathrm{Op}\left(\mathcal{M}_{p}^{\prime}\left(\mathbb{H}_{n}\right)\right)$, порожденной интегральными операторами $(14)$ с ядрами из $\mathcal{C}_{p}^{\prime}\left(\mathbb{H}_{n}\right)$.

Топологическое тензорное произведение $L_{p}(X, \mu) \otimes L_{p}(Y, v)$ весовых пространств $L_{p}(X, \mu)$ и $L_{p}(Y, v)$ определяется как замыкание в $L_{p}(X \times Y, \mu \otimes v)$ алгебраического тензорного произведения

$$
L_{p}(X, \mu) \odot L_{p}(Y, v)=\left\{\sum_{i=1}^{j} f_{i} g_{i}: f_{i} \in L_{p}(X, \mu), g_{i} \in L_{p}(Y, v), j \in \mathbb{N}\right\} .
$$

ISSN 2587-6325. Математ. физика и компьютер. моделирование. 2018. Т. 21. № 3 
Поскольку $L_{p}(X, \mu) \odot L_{p}(Y, \boldsymbol{v})$ плотно в $L_{p}(X \times Y, \mu \otimes v)$, то $L_{p}(X, \mu) \otimes L_{p}(Y, v)$ совпадает с $L_{p}(X \times Y, \mu \otimes v)$. Топологическое тензорное произведение $\mathfrak{A} \otimes \mathfrak{B}$ банаховых алгебр $\mathfrak{A} \subset \mathcal{L}\left(L_{p}(X, \mu)\right)$ и $\mathfrak{B} \subset \mathcal{L}\left(L_{p}(Y, \boldsymbol{v})\right)$ определяется как замыкание в $\mathcal{L}\left(L_{p}(X \times Y, \boldsymbol{\mu} \otimes \boldsymbol{v})\right)$ алгебраического тензорного произведения

$$
\mathfrak{A} \odot \mathfrak{B}=\left\{\sum_{i=1}^{j} R_{i} \otimes S_{i}: R_{i} \in \mathfrak{A}, S_{i} \in \mathfrak{B}, j \in \mathbb{N}\right\},
$$

где $R_{i} \otimes S_{i}$ - непрерывное продолжение оператора $R_{i} \odot S_{i}$, ограниченного в линеале $L_{p}(X, \mu) \odot L_{p}(Y, v)$, на банахово пространство $L_{p}(X \times Y, \mu \otimes v)$ [7, с. 110].

Идеал компактных в пространстве $L_{p}\left(\mathbb{S}_{n}\right)$ операторов обозначим $\mathcal{K}_{p}\left(\mathbb{S}_{n}\right)$. Следующее утверждение доказывается по схеме доказательства леммы 1.1 из [4].

Лемма 2. $\mathfrak{V}_{p}^{\prime}\left(\mathbb{H}_{n}\right)=\mathcal{V}_{p}\left(\mathbb{R}_{+}, r^{2 n+1}\right) \otimes \mathcal{K}_{p}\left(\mathbb{S}_{n}\right)$.

Пример 1. Построим интегральный оператор с однородным ядром компактного типа. Определенная на $\mathbb{H}_{n} \times \mathbb{H}_{n}$ функция

$$
\kappa(x, y)=\frac{1}{\|x\|^{2 n+2}+\|y\|^{2 n+2}} \xi\left(\delta_{\|x\|}^{-1}(x), \delta_{\|y\|}^{-1}(y)\right), \quad \xi \in L_{\infty}\left(\mathbb{S}_{n} \times \mathbb{S}_{n}\right),
$$

является однородной степени $(-2 n-2)$ (см. (5)). Проверим для функции к выполнение условий $\varkappa_{1}(\kappa), \varkappa_{2}(\kappa)<\infty$, (см. (6)-(7)). Рассмотрим функцию

$$
\kappa_{1}(\sigma)=\int_{\mathbb{H}_{n}} \frac{1}{\|x\|^{\frac{2 n+2}{p^{\prime}}}+\|x\|^{(2 n+2)\left(\frac{1}{p^{\prime}}+1\right)}}\left|\xi\left(\delta_{\|x\|}^{-1}(x), \sigma\right)\right| d x, \quad \sigma \in \mathbb{S}_{n} .
$$

Справедлива оценка

$$
\forall \sigma \in \mathbb{S}_{n}: \quad \kappa_{1}(\sigma) \leqslant\|\xi\|_{L_{\infty}\left(\mathbb{S}_{n} \times \mathbb{S}_{n}\right)} \int_{\mathbb{H}_{n}} \frac{d x}{\|x\|^{\frac{2 n+2}{p^{\prime}}}+\|x\|^{(2 n+2)\left(\frac{1}{p^{\prime}}+1\right)}} .
$$

Интеграл в правой части неравенства сходится в силу утверждения 3 леммы, доказанной в [3], и, следовательно,

$$
\varkappa_{1}(\mathrm{\kappa})=\left\|\mathrm{\kappa}_{1}\right\|_{L_{\infty}\left(\mathbb{S}_{n}\right)}<\infty .
$$

Аналогично проверяется выполнение условия $\varkappa_{2}(\kappa)<\infty$. Таким образом, $к \in \mathcal{M}_{p}\left(\mathbb{H}_{n}\right)$.

Применяя к функции к отображение $\widetilde{Q}$ (см. (13)), получим

$$
\widetilde{Q}(\kappa)(r, \rho, s, \sigma)=\rho^{2 n+1} \frac{1}{r^{2 n+2}+\rho^{2 n+2}} \xi(s, \sigma), \quad r, \rho \in \mathbb{R}_{+}, s, \sigma \in \mathbb{S}_{n} .
$$

Функцию $\widetilde{Q}(\kappa)$ представим в виде $\widetilde{Q}(\kappa)=\kappa_{0} \xi$, где

$$
\kappa_{0}(r, \rho)=\rho^{2 n+1} \frac{1}{r^{2 n+2}+\rho^{2 n+2}} .
$$

Несложно видеть, что функция к

$$
\alpha\left(\kappa_{0}\right)=\int_{0}^{\infty}\left|\kappa_{0}(1, \rho)\right| \rho^{-\frac{1}{p}} d \rho=\int_{0}^{\infty} \frac{1}{\rho^{1-\frac{2 n+2}{p^{\prime}}}+\rho^{1+\frac{2 n+2}{p}}} d \rho
$$


(см. (19)) сходится и, следовательно, $\kappa_{0} \in \mathcal{A}_{p}\left(\mathbb{R}_{+}, r^{2 n+1}\right)$. Таким образом, мы установили, что $\widetilde{Q}(\kappa) \in \mathcal{C}_{p}^{\prime}\left(\mathbb{H}_{n}\right), \kappa \in \mathcal{C}_{p}\left(\mathbb{H}_{n}\right)$, а оператор вида (11) с ядром к

$$
\left(K_{\mathrm{K}} f\right)(x)=\int_{\mathbb{H}_{n}} \frac{1}{\|x\|^{2 n+2}+\|y\|^{2 n+2}} \xi\left(\delta_{\|x\|}^{-1}(x), \delta_{\|y\|}^{-1}(y)\right) f(y) d y
$$

является ограниченным в $L_{p}\left(\mathbb{H}_{n}\right)$ интегральным оператором с однородным ядром компактного типа.

\section{5. Символическое исчисление и условие обратимости для $\mathfrak{V}_{p}^{+}\left(\mathbb{H}_{n}\right)$}

Рассмотрим сверточное представление алгебры $\mathfrak{V}_{p}^{+}\left(\mathbb{H}_{n}\right)$. С помощью изометрического изоморфизма $u_{p}^{2 n+1}: L_{p}\left(\mathbb{R}_{+}, r^{2 n+1}\right) \rightarrow L_{p}(\mathbb{R})$ вида

$$
\left(u_{p}^{2 n+1} f\right)(t)=e^{\frac{2 n+2}{p} t} f\left(e^{t}\right), \quad t \in \mathbb{R}
$$

и конструкции (15) определим изоморфизм подобия банаховых алгебр

$$
\hat{u}_{p}^{2 n+1}: \mathcal{L}\left(L_{p}\left(\mathbb{R}_{+}, r^{2 n+1}\right)\right) \rightarrow \mathcal{L}\left(L_{p}(\mathbb{R})\right) .
$$

Замкнутую подалгебру банаховой алгебры $\mathcal{L}\left(L_{p}(\mathbb{R})\right)$, порожденную операторами аддитивной свертки

$$
\left(C_{c} f\right)(t)=\int_{-\infty}^{\infty} c(t-\tau) f(\tau) d \tau, \quad c \in L_{1}(\mathbb{R})
$$

обозначим $V_{p}(\mathbb{R})$.

Непосредственными выкладками доказывается, что ограничение $\hat{u}_{p}^{2 n+1}$ на $\mathcal{V}_{p}\left(\mathbb{R}_{+}, r^{2 n+1}\right)$ задает изоморфизм

$$
\mathbf{u}_{p}^{2 n+1}: \mathcal{V}_{p}\left(\mathbb{R}_{+}, r^{2 n+1}\right) \rightarrow V_{p}(\mathbb{R}),
$$

причем $\mathbf{u}_{p}^{2 n+1}\left(A_{a}\right)=C_{c}$, где $A_{a}$ - оператор вида (20), а $C_{c}$ - оператор свертки с ядром

$$
c(t)=e^{\frac{2 n+2}{p} t} a\left(e^{t}, 1\right) .
$$

Отметим, что ограничение $\widehat{Q}$ (см. (16)) на алгебру $\mathfrak{V}_{p}\left(\mathbb{H}_{n}\right)$ задает изоморфизм $\mathrm{Q}: \mathfrak{V}_{p}\left(\mathbb{H}_{n}\right) \rightarrow \mathfrak{V}_{p}^{\prime}\left(\mathbb{H}_{n}\right)$ и рассмотрим оператор

$$
\mathbf{u}_{p}^{2 n+1} \otimes I: \mathcal{V}_{p}\left(\mathbb{R}_{+}, r^{2 n+1}\right) \otimes \mathcal{K}_{p}\left(\mathbb{S}_{n}\right) \rightarrow V_{p}(\mathbb{R}) \otimes \mathcal{K}_{p}\left(\mathbb{S}_{n}\right) .
$$

Учитывая лемму 2, построим оператор

$$
\left(\mathbf{u}_{p}^{2 n+1} \otimes I\right) \mathbf{Q}: \mathfrak{V}_{p}\left(\mathbb{H}_{n}\right) \rightarrow V_{p}(\mathbb{R}) \otimes \mathcal{K}_{p}\left(\mathbb{S}_{n}\right),
$$

порождающий изоморфизм

$$
\mathbf{U}_{p}: \mathfrak{V}_{p}^{+}\left(\mathbb{H}_{n}\right) \rightarrow\left(V_{p}(\mathbb{R}) \otimes \mathcal{K}_{p}\left(\mathbb{S}_{n}\right)\right)^{+}
$$


Изоморфизм $\mathbf{U}_{p}$ мы будем называть сверточным представлением алгебры $\mathfrak{V}_{p}^{+}\left(\mathbb{H}_{n}\right)$.

Построим символ для операторов из алгебры $\left(V_{p}(\mathbb{R}) \otimes \mathcal{K}_{p}\left(\mathbb{S}_{n}\right)\right)^{+}$. Пусть $\mathbb{R}-$ компактификация пространства $\mathbb{R}$ бесконечно удаленной точкой $\infty ; \mathfrak{A}-$ произвольная банахова алгебра, тогда через $C(\dot{\mathbb{R}}, \mathfrak{A})$ обозначим банахову алгебру непрерывных отображений $\mathbb{R}$ в $\mathfrak{A}$ с равномерной топологией. Будем полагать, что

$$
C_{0}(\dot{\mathbb{R}}, \mathfrak{A})=\{f \in C(\dot{\mathbb{R}}, \mathfrak{A}): f(\infty)=0\} .
$$

Известно, что сопоставление оператору свертки $C_{c} \in V_{p}(\mathbb{R})$ преобразования Фурье его ядра задает непрерывный мономорфизм $\phi_{p}: V_{p}(\mathbb{R}) \rightarrow C_{0}(\dot{\mathbb{R}}, \mathbb{C})[9]$.

Рассмотрим оператор

$$
\widetilde{\Phi}_{p}: V_{p}(\mathbb{R}) \odot \mathcal{K}_{p}\left(\mathbb{S}_{n}\right) \rightarrow C_{0}\left(\dot{\mathbb{R}}, \mathcal{K}_{p}\left(\mathbb{S}_{n}\right)\right),
$$

определяемый по формуле

$$
\left(\widetilde{\Phi}_{p}\left(\sum_{i=1}^{j} R_{i} \otimes S_{i}\right)\right)(t)=\sum_{i=1}^{j}\left(\phi_{p}\left(R_{i}\right)\right)(t) S_{i}, \quad t \in \mathbb{R} .
$$

Оператор $\widetilde{\Phi}_{p}$ может быть непрерывно продолжен до оператора

$$
\Phi_{p}: V_{p}(\mathbb{R}) \otimes \mathcal{K}_{p}\left(\mathbb{S}_{n}\right) \rightarrow C_{0}\left(\dot{\mathbb{R}}, \mathcal{K}_{p}\left(\mathbb{S}_{n}\right)\right)
$$

Легко видеть, что алгебра операторнозначных функций

$$
C_{0, p}\left(\dot{\mathbb{R}}, \mathcal{K}_{p}\left(\mathbb{S}_{n}\right)\right)=\Phi_{p}\left(V_{p}(\mathbb{R}) \otimes \mathcal{K}_{p}\left(\mathbb{S}_{n}\right)\right)
$$

с нормой

$$
\|f\|_{C_{0, p}\left(\dot{\mathbb{R}}, \mathcal{K}_{p}\left(\mathbb{S}_{n}\right)\right)}=\left\|\Phi_{p}^{-1} f\right\|_{\mathcal{L}\left(L_{p}\left(\mathbb{R} \times \mathbb{S}_{n}\right)\right)}
$$

является банаховой.

Ограничение оператора $\Phi_{p}$ на образ порождает изоморфизм

$$
\boldsymbol{\Phi}_{p}:\left(V_{p}(\mathbb{R}) \otimes \mathcal{K}_{p}\left(\mathbb{S}_{n}\right)\right)^{+} \rightarrow C_{0, p}^{+}\left(\dot{\mathbb{R}}, \mathcal{K}_{p}\left(\mathbb{S}_{n}\right)\right),
$$

который мы будем называть символом для операторов из $\left(V_{p}(\mathbb{R}) \otimes \mathcal{K}_{p}\left(\mathbb{S}_{n}\right)\right)^{+}$.

Лемма 3. Пусть $C \in\left(V_{p}(\mathbb{R}) \otimes \mathcal{K}_{p}\left(\mathbb{S}_{n}\right)\right)^{+}$. Тогда для того, итобы оператор $C$ был обратим в алгебре $\left(V_{p}(\mathbb{R}) \otimes \mathcal{K}_{p}\left(\mathbb{S}_{n}\right)\right)^{+}$, необходимо и достаточно, итобы его символ $\mathbf{\Phi}_{p}(C)$ был обратим в алгебре $\left.C_{0}^{+}\left(\dot{\mathbb{R}}, \mathcal{K}_{p}\left(\mathbb{S}_{n}\right)\right)\right)$.

Доказательство. Пусть $\mathrm{L}(m, \mathbb{C})-$ банахова алгебра $m \times m$ матриц над полем $\mathbb{C}$. Из того, что $\boldsymbol{\Phi}_{p}-$ изоморфизм алгебр, следует, что оператор $C$ обратим в алгебре $\left(V_{p}(\mathbb{R}) \otimes \mathcal{K}_{p}\left(\mathbb{S}_{n}\right)\right)^{+}$тогда и только тогда, когда его символ $\boldsymbol{\Phi}_{p}(C)$ обратим в алгебре $C_{0, p}^{+}\left(\dot{\mathbb{R}}, \mathcal{K}_{p}\left(\mathbb{S}_{n}\right)\right)$. Обратимость же символа в $C_{0, p}^{+}\left(\dot{\mathbb{R}}, \mathcal{K}_{p}\left(\mathbb{S}_{n}\right)\right)$ равносильна его обратимости в $C_{0}^{+}\left(\dot{\mathbb{R}}, \mathcal{K}_{p}\left(\mathbb{S}_{n}\right)\right)$. Данное утверждение выводится из результата И.Б. Симоненко о том, что обратимость матричнозначной функции в аналогичной банаховой алгебре $C_{0, p}^{+}(\dot{\mathbb{R}}, \mathrm{L}(m, \mathbb{C}))$ равносильна ее обратимости в $C_{0}^{+}(\dot{\mathbb{R}}, \mathrm{L}(m, \mathbb{C}))($ см. $[9$, с. $307 ; 10$, с. 46$])$ и того факта, что индуктивный предел $\mathrm{L}(\infty, \mathbb{C})$ плотно вложен в $\mathcal{K}_{p}\left(\mathbb{S}_{n}\right)$. Лемма доказана. 
Символ для операторов из алгебры $\mathfrak{V}_{p}^{+}\left(\mathbb{H}_{n}\right)$ построим с помощью ее сверточного представления $\mathbf{U}_{p}$ (см. (22)) и символа $\boldsymbol{\Phi}_{p}($ см. $(23))$ для операторов из алгебры $\left(V_{p}(\mathbb{R}) \otimes\right.$ $\left.\otimes \mathcal{K}_{p}\left(\mathbb{S}_{n}\right)\right)^{+}$

$$
\Upsilon_{p}=\Phi_{p} \mathbf{U}_{p} .
$$

Из леммы 3 вытекает следующий критерий обратимости операторов из $\mathfrak{V}_{p}^{+}\left(\mathbb{H}_{n}\right)$.

Теорема 2. Пусть $K \in \mathfrak{V}_{p}^{+}\left(\mathbb{H}_{n}\right)$. Тогда для того, итобы оператор $K$ был обратим в алгебре $\mathfrak{V}_{p}^{+}\left(\mathbb{H}_{n}\right)$, необходимо и достаточно, чтобы его символ $\Upsilon_{p}(K)$ был обратим в алгебре $C_{0}^{+}\left(\dot{\mathbb{R}}, \mathcal{K}_{p}\left(\mathbb{S}_{n}\right)\right)$ ).

Таким образом, проверка обратимости произвольного оператора $K \in \mathfrak{V}_{p}^{+}\left(\mathbb{H}_{n}\right)$ сводится к проверке обратимости параметризованного $\dot{\mathbb{R}}$ семейства операторов более простого вида.

Пример 2. В алгебре $\mathfrak{V}_{p}^{+}\left(\mathbb{H}_{n}\right)$ рассмотрим оператор

$$
K=\lambda I+K_{\mathrm{K}}
$$

где $K_{\mathrm{\kappa}}$ - оператор (21) из примера $1, \lambda \in \mathbb{C}$. Воспользуемся конструкцией символического исчисления для алгебры $\mathfrak{V}_{p}^{+}\left(\mathbb{H}_{n}\right)$ и вычислим символ оператора $K$. Тогда

$$
\Upsilon_{p}(K)(\eta)=\lambda I_{L_{p}\left(\mathbb{S}_{n}\right)}+\Theta(\eta) T
$$

где $\eta \in \dot{\mathbb{R}}, T \in \mathcal{K}_{p}\left(\mathbb{S}_{n}\right)$ - компактный оператор вида

$$
(T f)(s)=\int_{\mathbb{S}_{n}} \xi(s, \sigma) f(\sigma) d \sigma
$$

с ядром $\xi \in L_{\infty}\left(\mathbb{S}_{n} \times \mathbb{S}_{n}\right), \Theta(\eta)-$ преобразование Фурье функции

$$
\frac{e^{\frac{2 n+2}{p} t}}{e^{(2 n+2) t}+1} \in L_{1}(\mathbb{R}) \text {. }
$$

Итак, проверка обратимости оператора $K=\lambda I+K_{\mathrm{\kappa}}\left(\in \mathfrak{V}_{p}^{+}\left(\mathbb{H}_{n}\right)\right)$ сводится к проверке обратимости параметризованного $\mathbb{R}$ семейства операторов более простого вида из алгебры $\mathcal{K}_{p}^{+}\left(\mathbb{S}_{n}\right)$

\section{СПИСОК ЛИТЕРАТУРЫ}

1. Авсянкин, О. Г. О С*-алгебре, порожденной многомерными интегральными операторами с однородными ядрами и операторами мультипликативного сдвига / О. Г. Авсянкин // Доклады Академии наук. - 2008. - Т. 419, вып. 6. - С. 727-728.

2. Бесов, О. В. Интегральные представления функций и теоремы вложения / О. В. Бесов, В. П. Ильин, С. М. Никольский. - М. : Наука, 1975. - 480 с.

3. Денисенко, В. В. Об ограниченности интегральных операторов с однородными ядрами на группе Гейзенберга с нормой Кораньи / В. В. Денисенко, В. М. Деундяк // Известия высших учебных заведений. Северо-Кавказский регион. Естественные науки. 2017. - № 3-1. - С. 21-27.

4. Деундяк, В. М. Многомерные интегральные операторы с однородными ядрами компактного типа и мультипликативно слабо осциллирующими коэффициентами / В. М. Деундяк // Мат. заметки. - 2010. - Т. 87, вып. 5. - С. 704-720. 
5. Деундяк, В. М. Об ограниченности и фредгольмовости интегральных операторов с анизотропно однородными ядрами компактного типа и переменными коэффициентами / В. М. Деундяк, Е. И. Мирошникова // Изв. вузов. Математика. - 2012. - № 7 . C. 3-17.

6. Крейн, С. Г. Интерполяция линейных операторов / С. Г. Крейн, Ю. И. Петунин, Е. М. Семенов. - M. : Наука, 1978. - 400 с.

7. Люстерник, Л. А. Краткий курс функционального анализа / Л. А. Люстерник, В. И. Соболев. - M. : Наука, 1982. - 270 с.

8. Симоненко, И. Б. Новый общий метод исследования линейных операторных уравнений типа сингулярных интегральных уравнений. II / И. Б. Симоненко // Изв. АН СССР. Сер. мат. - 1965. - Т. 29, вып. 4. - С. 757-782.

9. Симоненко, И. Б. Операторы типа свертки в конусах / И. Б. Симоненко // Мат. сб. - 1967. - Т. 74, № 2. - С. 298-313.

10. Симоненко, И. Б. Локальный метод в теории инвариантных относительно сдвига операторов и их огибающих / И. Б. Симоненко. - Ростов н/Д : Изд-во ЦВВР, 2007. - 120 с.

11. An Introduction to the Heisenberg Group and the Sub-Riemannian Isoperimetric Problem / L. Capogna, D. Danielli, S. D. Pauls, J. Tyson. - Basel : Birkhauser, 2007. - 224 p.

12. Chirikjian, G. S. Engineering applications of noncommutative harmonic analysis: with emphasis on rotation and motion groups / G. S. Chirikjian, A. B. Kyatkin. - Boca Raton : CRC Press, 2001. - 698 p.

13. Deundyak, V. M. Convolution Operators with Weakly Oscillating Coeffcients in Hilbert Moduli on Groups and Applications / V. M. Deundyak // Journal of Mathematical Sciences. 2015. - Vol. 208, iss. 1. - P. 100-108.

14. Karapetiants, N. Equations with Involutive Operators / N. Karapetiants, S. Samko. Boston: Birkhauser, 2001. - 642 p.

15. Kisil, V. V. Symmetry, geometry, and quantization with hypercomplex numbers / V. V. Kisil // Geometry, Integrability and Quantization. - 2017. - Vol. 18. - P. 11-76.

16. Krantz, S. G. Explorations in harmonic analysis: with applications to complex function theory and the Heisenberg group / S. G. Krantz. - Boston : Birkhauser, 2009. - 360 p.

\section{REFERENCES}

1. Avsyankin O.G. O C*-algebre, porozhdennoy mnogomernymi integralnymi operatorami s odnorodnymi yadrami i operatorami multiplikativnogo sdviga [On the $\mathrm{C}^{*}$-Algebra Generated by Multidimensional Integral Operators with Homogeneous Kernels and Multiplicative Shift Operators]. Doklady Akademii nauk [Doklady Mathematics], 2008, vol. 419, iss. 6, pp. 727-728.

2. Besov O.V., Ilin V.P., Nikolskiy S.M. Integralnye predstavleniya funktsiy i teoremy vlozheniya [Integral Representations of Functions and Imbedding Theorems]. Moscow, Nauka Publ., 1975. 480 p.

3. Denisenko V.V., Deundyak V.M. Ob ogranichennosti integralnykh operatorov s odnorodnymi yadrami na gruppe Geyzenberga s normoy Korani [On the Boundedness of Integral Operators with Homogeneous Kernels on the Heisenberg Group with Koranyi Norm]. Izvestiya vysshikh uchebnykh zavedeniy. Severo-Kavkazskiy region. Estestvennye nauki, 2017, no. 3-1, pp. 21-27.

4. Deundyak V.M. Mnogomernye integralnye operatory s odnorodnymi yadrami kompaktnogo tipa i multiplikativno slabo ostsilliruyushchimi koeffitsientami [Multidimensional Integral Operators with Homogeneous Kernels of Compact Type and Multiplicatively Weakly Oscillating Coefficients]. Mat. zametki [Mathematical Notes], 2010, vol. 87, iss. 5, pp. 704-720.

5. Deundyak V.M., Miroshnikova E.I. Ob ogranichennosti i fredgolmovosti integralnykh operatorov s anizotropno odnorodnymi yadrami kompaktnogo tipa i peremennymi koeffitsientami [The Boundedness and the Fredholm Property of Integral Operators with Anisotropically Homogeneous Kernels of Compact Type and Variable Coefficients]. Izv. vuzov. Matematika [Russian Mathematics], 2012, no. 7, pp. 3-17. 
6. Kreyn S.G., Petunin Yu.I., Semenov E.M. Interpolyatsiya lineynykh operatorov [Interpolation of Linear Operators]. Moscow, Nauka Publ., 1978. 400 p.

7. Lyusternik L.A., Sobolev V.I. Kratkiy kurs funktsionalnogo analiza [Brief Course of Functional Analysis]. Moscow, Nauka Publ., 1982. 270 p.

8. Simonenko I.B. Novyy obshchiy metod issledovaniya lineynykh operatornykh uravneniy tipa singulyarnykh integralnykh uravneniy. II [A New General Method of Investigating Linear Operator Equations of Singular Integral Equation Type. II]. Izv. AN SSSR. Ser. mat. [Izvestiya: Mathematics], 1965, vol. 29, iss. 4, pp. 757-782.

9. Simonenko I.B. Operatory tipa svertki v konusakh [Operators of Convolution Type in Cones]. Mat. sb. [Sbornik: Mathematics], 1967, vol. 74, no. 2, pp. 298-313.

10. Simonenko I.B. Lokalnyy metod v teorii invariantnykh otnositelno sdviga operatorov $i$ ikh ogibayushchikh [The Local Method in the Theory of Shift-Invariant Operators and Their Envelopes]. Rostov-on-Don, Izd-vo TsVVR Publ., 2007. 120 p.

11. Capogna L., Danielli D., Pauls S.D., Tyson J. An Introduction to the Heisenberg Group and the Sub-Riemannian Isoperimetric Problem. Basel, Birkhauser, 2007. 224 p.

12. Chirikjian G.S., Kyatkin A.B. Engineering applications of noncommutative harmonic analysis: with emphasis on rotation and motion groups. Boca Raton, CRC Press, 2001. 698 p.

13. Deundyak V.M. Convolution Operators with Weakly Oscillating Coeffcients in Hilbert Moduli on Groups and Applications. Journal of Mathematical Sciences, 2015, vol. 208, iss. 1, pp. 100-108.

14. Karapetiants N., Samko S. Equations with Involutive Operators. Boston, Birkhauser, 2001. 642 p.

15. Kisil V.V. Symmetry, Geometry, and Quantization with Hypercomplex Numbers. Geometry, Integrability and Quantization, 2017, vol. 18, pp. 11-76.

16. Krantz S.G. Explorations in harmonic analysis: with applications to complex function theory and the Heisenberg group. Boston, Birkhauser, 2009. 360 p.

\section{THE INVERTIBILITY OF INTEGRAL OPERATORS WITH HOMOGENEOUS KERNELS OF COMPACT TYPE ON THE HEISENBERG GROUP}

\section{Victor Vladimirovich Denisenko}

Postgraduate Student, Department of Algebra and Discrete Mathematics, Southern Federal University, Vorovich Institute of Mathematics, Mechanics and Computer Science vct.dns@gmail.com Milchakova St., 8a, 344090 Rostov-on-Don, Russian Federation

\section{Vladimir Mikhaylovich Deundyak}

Candidate of Physical and Mathematical Sciences, Associate Professor, Department of Algebra and Discrete Mathematics,

Southern Federal University, Vorovich Institute of Mathematics, Mechanics and Computer Science vl.deundyak@gmail.com Milchakova St., 8a, 344090 Rostov-on-Don, Russian Federation; Senior Researcher, Specvuzavtomatika Research Institute Gazetny lane, 51, 344002 Rostov-on-Don, Russian Federation

Abstract. Let $\mathbb{C}^{n}$ be a $n$-dimensional complex coordinate space and let $\mathbb{R}$ be a set of real numbers. The Heisenberg group is a set $\mathbb{H}_{n}=\mathbb{C}^{n} \times \mathbb{R}$ with the 
binary operation

$$
(z, a)(w, b)=(z+w, a+b+2 \operatorname{Im}(z \cdot w)), \quad(z, a),(w, b) \in \mathbb{H}_{n} .
$$

The group under consideration is endowed with a family of dilations

$$
\delta_{r}(z, a)=\left(r z, r^{2} a\right), \quad r \in \mathbb{R}_{+}, \quad(z, a) \in \mathbb{H}_{n},
$$

and is equipped with the Koranyi norm

$$
\|(z, a)\|=\left(|z|^{4}+a^{2}\right)^{\frac{1}{4}}, \quad(z, a) \in \mathbb{H}_{n} .
$$

This norm allows us to define the notion of the unit ball on the Heisenberg group

$$
\mathbb{S}_{n}=\left\{x \in \mathbb{H}_{n}:\|x\|=1\right\} .
$$

The transformation of Cartesian coordinates on the Heisenberg group $x \in \mathbb{H}_{n} \backslash\{(\mathbf{0}, 0)\}$ to spherical coordinates $(r, s) \in \mathbb{R}_{+} \times \mathbb{S}_{n}$ is defined by

$$
r=\|x\|, \quad s=\delta_{\|x\|}^{-1}(x) .
$$

The function $k: \mathbb{H}_{n} \times \mathbb{H}_{n} \rightarrow \mathbb{C}$ is said to be homogeneous of degree $m$ if it satisfies the condition of homogeneity

$$
\forall \gamma \in \mathbb{R}_{+}, \quad \forall x, y \in \mathbb{H}_{n}: \quad k\left(\delta_{\gamma}(x), \delta_{\gamma}(y)\right)=\gamma^{m} k(x, y) .
$$

This paper is concerned with the study of linear integral operators on the Heisenberg group of the form

$$
\left(K_{k} f\right)(x)=\int_{\mathbb{H}_{n}} k(x, y) f(y) d y,
$$

where function $k$ is an element of the special Banach space $\mathcal{M}_{p}\left(\mathbb{H}_{n}\right)$ of homogeneous $(-2 n-2)$ degree functions. It is claimed that operator under consideration is bounded in the space $L_{p}\left(\mathbb{H}_{n}\right)$, where $1<p<\infty$.

A new class $\mathcal{C}_{p}\left(\mathbb{H}_{n}\right) \subset \mathcal{M}_{p}\left(\mathbb{H}_{n}\right)$ of homogeneous kernels of compact type is introduced. The main object of the research is the unitary Banach algebra $\mathfrak{V}_{p}^{+}\left(\mathbb{H}_{n}\right)$ generated by integral operators with $\mathcal{C}_{p}\left(\mathbb{H}_{n}\right)$ kernels. It should be pointed out that spherical coordinate system on the Heisenberg group plays a significant role in construction of the $\mathcal{C}_{p}\left(\mathbb{H}_{n}\right)$ class.

The convolutional representation of the unitary Banach algebra $\mathfrak{V}_{p}^{+}\left(\mathbb{H}_{n}\right)$ is constructed using the technique of tensor products. This representation makes it possible to define the symbol for integral operators in $\mathfrak{V}_{p}^{+}\left(\mathbb{H}_{n}\right)$ algebra and formulate the necessary and sufficient conditions for invertibility of these operators in terms of their symbol.

Key words: Heisenberg group, linear integral operators, operators with homogeneous kernels, convolutional representation, symbolic calculus, invertibility of operators. 